\title{
Influencia de las inteligencias múltiples en la elección de carreras profesionales en estudiantes de cuarto y quinto de secundaria
}

\author{
Influence of multiple intelligences in the choice of professionals careers in students \\ of fourth and fifth grades of secondary education
}

Víctor Elías Ríos Cubas', Luz María Supo Zapata²

Universidad Continental

\section{RESUMEN}

Objetivos: Determinar la influencia de las inteligencias múltiples en la elección de carreras profesionales en estudiantes del cuarto y quinto de secundaria del sistema educativo peruano. Métodos: La investigación es de alcance descriptivo correlacional con diseño transversal. En la recolección de datos se utilizaron los instrumentos, test de inteligencias múltiples e inventario de intereses profesionales de Angellini. La población investigada estuvo conformada por 13459 alumnos con un nivel de confianza del $99 \%$ y $1,5 \%$ de error para cuarto grado y $1 \%$ de error para el quinto. Resultados: Se encontró que los estudiantes tienen mayor puntuación en las inteligencias intrapersonal $(15,8 \%)$, lógico-matemática $(14,1 \%)$, naturalista o ecológica $(13,7 \%)$ y musical o rítmica $(12,9 \%)$ haciendo un total del 56,5\%, y menor puntuación en las demás inteligencias como: verbal-lingüística, visual-espacial, kinestésica, interpersonal, humanista, religiosa-espiritual y sexual, las cuales representan un total de $43,5 \%$. Por otro lado en relación a las áreas vocacionales se destacan las de ciencias físicas $(34,6 \%)$ y de ciencias biológicas $(16,8 \%)$ haciendo un total de $51,4 \%$, mientras que el $48,6 \%$ se encuentra distribuido en las otras áreas. En la población de mujeres se destacaron las Inteligencias intrapersonal (18,9\%), naturalista o ecológica $(14,2 \%)$ y musical o rítmica $(13,8 \%)$ con un total de $46,9 \%$ y el $53,1 \%$ están distribuidos en otras inteligencias. En el caso de varones se destacaron las inteligencias lógico-matemática (21,3\%), naturalista o ecológica $(13,2 \%)$ e intrapersonal

$(12,2 \%)$ con un total de $46,7 \%$, y el $53,3 \%$ están distribuidas en otras inteligencias. Conclusiones: Existe relación entre las inteligencias múltiples y la elección del área de las carreras vocacionales, lo cual es importante para el desarrollo profesional.

Palabras clave: Inteligencias múltiples, orientación vocacional, elección, habilidades.

1 Lic. en Psicología, Mg. en recursos humanos, Mg. en desarrollo de habilidades y competencias, candidato a doctor en Psicología, consultor senior y gerente general de la Consultora "Liderando Kambios".

2 Lic. en Estadística, candidata a magister en administración de negocios, docente investigadora de la Universidad Continental. 


\section{ABSTRACT}

Objectives: To determine the multiple intelligences' influence in choosing professional careers in the fourth and fifth students secondary on Peruvian education system. Methods: The research has correlational descriptive scope and crosssectional design. In the data collection was used instruments likethe multipleintelligences test and the Angellini professional interests' inventory. The research population has been formed by 13459 students using a statistical confidence level of $99 \%$ and $1.5 \%$ error in fourth grade and $1 \%$ error in fifth grade. Results: We found that students have more punctuation in intrapersonal intelligences (15.8\%), logical-mathematical (14.1\%), naturalistic or ecological (13.7\%) and musical or rhythmic (12.9\%) resulting in a total of $56.5 \%$ and less punctuation in the rest of the intelligences like: verbal-linguistic, visual-spatial, kinesthetic, interpersonal, humanistic, religious-spiritual and sexual, which represent a total of $43.5 \%$. On the other hand in relation to vocational areas are highlighted the physical sciences (34.6\%) and biological sciences $(16.8 \%$ ) resulting in a total of $51.4 \%$, while the $48.6 \%$ is distributed in the other areas. In women population are emphasized the intrapersonal intelligences (18.9\%), naturalistic or ecological (14.2\%) and musical or rhythmic (13.8\%) with a total of $46.9 \%$ and $53.1 \%$ are distributed in other intelligences. For men are emphasized the logical-mathematical intelligences (21.3\%), naturalistic or ecological (13.2\%) and intrapersonal $(12.2 \%)$ resulting in a total of $46.7 \%$, and $53.3 \%$ are distributed in other intelligences. Conclusions: There is a relation between multiple intelligences and the choice of the vocational careers area, which is important for the professional development.

Keywords: Multiple intelligences, vocational counseling, choice, skills.

\section{INTRODUCCIÓN}

Esta investigación se sitúa en un contexto en el cual los jóvenes alumnos de los últimos grados deeducación secundaria deben elegir una carrera profesional. Hacerlo en estos tiempos precisa mucho más la necesidad de advertir, de manera especial, cómo inciden las distintas inteligencias, planteadas por Gardner, en la decisión vocacional. Y es que la elección de una carrera profesional en los jóvenes es compleja y necesita de antecedentes importantes como la personalidad, el entorno familiar-social y el tipo de aprendizaje (1), los cuales se han acumulado a lo largo de su vida personal y académica (2).

Responder a esta complejidad previene deserciones y frustraciones académicas, así como la incertidumbre por el futuro a elegir (3). Por lo tanto, es una temática recurrente en estos procesos, es la decisión de un estilo de vida (4), en la que los alumnos tendrán que desarrollar sus habilidades y adaptarse a diversos contextos, los cuales son propios de la carrera profesional elegida (5).

Aquellos jóvenes que elijan la carrera de Derecho, quienes aplicarán las leyes para defender derechos y deberes de las personas, tendrán, por ejemplo, un entorno cotidiano que les exija resolver casos en medio de reclamos y denuncias, situación que les permitirá desarrollar habilidades de tolerancia, vocación de servicio y equidad; en cambio, en aquellos jóvenes que elijan la carrera de Psicología, quienes tendrán un entorno de dificultades emocionales y conductuales, la situación les permitirá desarrollar otras habilidades: de observación, escucha activa, seguridad y equilibrio personal. Ambos ejemplos nos permite advertir que las distintas profesiones adoptan un estilo de vida de acuerdo con su quehacer cotidiano profesional y laboral, para lo cual cada carrera necesitará de determinadas habilidades en su personalidad.

En este escenario, los orientadores tendrán que resolver dudas y conflictos 
de los jóvenes (6), realizando un acompañamiento y orientación durante todo el proceso vocacional (7). Para ello, tendrán que reforzar aquellas conductas positivas orientadas al logro de objetivos y metas (8), promoviendo habilidades, actitudes y valores (9), con la finalidad de mantener el equilibrio de su personalidad y la consolidación de su identidad (10), y así desarrollar un perfil profesional (11) de acuerdo con las exigencias de las competencias profesionales del mercado laboral (5). En este sentido se deberá fortalecer y mejorar las cuatro dimensiones humanas: la dimensión I, relacionada con todo lo biológico-tangible; la dimensión II, con todo lo emocional-subjetivo; la dimensión III, con la interacción social; y la dimensión IV, con todo lo cognitivo-objetivo (12).

Mantener el equilibrio de las cuatro dimensiones humanas, entonces, es reconocer la diversidad de opciones que tienen los jóvenes para captar y aprender; lo cual es adecuado para su realización profesional (13). Es más, también debemos promover aquellos factores internos (14) que permitan tener consistencia en la elaboración de sus estrategias personales basadas en sus recursos psicológicos internos (15), y el desarrollo de conductas eficaces en la peculiaridad de su personalidad, utilizando para ello su capacidad de aprendizaje (16).

Uno de los factores internos que ayudan a determinar una adecuada elección profesional es precisamente la inteligencia (dimensión cognitiva-objetiva), la cual permite aprender experiencias y conocimientos, deliberar alternativas y decidir la opción que se ajuste a los intereses y necesidades personales, y determinaciones vocacionales profesionales (13). Gardner asume la inteligencia como un potencial biológico y psicológico, que permite procesar información activada por un entorno vivencial con la finalidad de resolver problemas o crear productos que sean valiosos en un contexto cultural (17). También plantea diversas formas de demostrar y desarrollar la inteligencia, que es materia de interés para nuestra investigación.

Pero es más, Gardner establece que existen diversas formas de demostrar y desarrollar la inteligencia para resolver problemas y asumir un estilo de aprendizaje acorde con el contexto. Estas diversas formas de inteligencia son las denominadas inteligencias múltiples, que para efecto de nuestro estudio consideramos once: La inteligencia lógico-matemática, la inteligencia lingüística, la inteligencia espacial, la inteligencia musical, la inteligencia corporal-kinestésica, la inteligencia interpersonal, la inteligencia intrapersonal, la inteligencia naturalista, la inteligencia espiritual, la inteligencia humanista y la inteligencia sexual.

Nuestra investigación toma a las inteligencias múltiples como una variable de influencia en la elección de carreras profesionales en los procesos de orientación vocacional (18), para determinar el desarrollo de intereses basados en el autoconocimiento y autoconcepto (19). En este sentido, diversos autores como Lemos y Thomas (15), Noronha (10), Godoy (18), Ríos (1) y Aisenson (5) realizaron investigaciones que muestran la correlación entre los intereses vocacionales, la inteligencia y la personalidad; demostrando que los cuestionarios vocacionales para elegir una carrera profesional no son lo suficientemente consistentes, por lo que es necesario desarrollar el nuevo paradigma de: evaluar la inteligencia y las diversas formas de aprender (21), para direccionar y precisar la decisión vocacional.

Por lo tanto, los jóvenes que decidan su carrera profesional deben ser conscientes de que desarrollarán un estilo de vida en sus futuras competencias profesionales y laborales, en la cual tendrán que demostrar las diversas formas de aprendizaje que han desarrollado durante su vida. En este ámbito, hay experiencias participativas prácticas que promueven una metodología pedagógica individual y grupal, en la que utilizan a las inteligencias múltiples para que los alumnos conozcan sus habilidades 
y preferencias, y así puedan definir su perfil profesional (22). Estas experiencias preliminares deben ser consideraras por los educadores, padres de familia, orientadores y los propios jóvenes, como una nueva perspectiva de elección de una carrera profesional y un estilo de vida para su futuro.

\section{MATERIAL Y METODOS}

La investigación fue de alcance descriptivo - correlacional; la recolección de datos se realizó a partir de una muestra constituida por 134 colegios $(46$ estatales y 88 particulares) del cuarto y quinto año de nivel secundario del departamento de Junín, evaluándose a un total de 13459 estudiantes a través de la aplicación de los

Tabla № 1: Relación de tipos de inteligencia.

\begin{tabular}{|c|c|}
\hline $\begin{array}{l}\text { Tipo de } \\
\text { inteligencia }\end{array}$ & Descripción de capacidades \\
\hline Verbal lingüística & Transmisión y comprensión de mensajes. \\
\hline Lógico matemático & Cálculo matemático, pensamiento numérico, razonamiento y resolución de problemas. \\
\hline Visual espacial & Reconocimiento y distinción de objetos en espacio y dimensiones. \\
\hline Kinestésica o corporal & Unir el cuerpo y la mente para lograr el desempeño físico. \\
\hline Musical o rítmica & Asociar y/o distinguir el estímulo sonoro artístico. \\
\hline Intrapersonal & Formar una imagen veraz y precisa de nosotros mismos. \\
\hline Interpersonal & Manejar relaciones humanas y la empatía. \\
\hline Naturalista & Distinguir y utilizar elementos del medio ambiente. \\
\hline Humanista & La búsqueda del bien común y el aprendizaje de experiencias. \\
\hline Religiosa espiritual & Afrontar y resolver problemas de significados y valores. \\
\hline Sexual & Identificación y autovaloración de su propio organismo. \\
\hline
\end{tabular}

Tabla N²: Inventario de áreas profesionales y carreras afines.

\begin{tabular}{ll}
\multicolumn{1}{c}{ Área } & \\
& \\
\hline Artístico & Arquitectura, diseñador gráfico, diseñador de modas, publicista. \\
Cálculo & Matemática, contador, banca y finanzas, comercio exterior, economía. \\
Ciencias biológicas & Medicina humana, biología, bioquímica y farmacia, enfermería, tecnología médica, \\
& nutricionista, odontología. \\
Ciencias físicas & Ingeniería mecánica, ingeniería civil, ingeniería eléctrica, ingeniería de sistemas e \\
& informática, ingeniería de minas, ingeniería ambiental, ingeniería industrial. \\
Literario & Periodista (cronista, corresponsal), escritor, idiomas. \\
Musical & Licenciatura en arte (música, danza clásica y contemporánea). \\
Organización burocrática & Asistente de gerencia, contabilidad, marketing, administración, ingeniería en \\
& agro negocios. \\
Persuasivo & Ciencias de la comunicación, derecho, diplomacia. \\
Servicio social humanit. & Psicología, trabajo social, pedagogía. \\
\hline
\end{tabular}


siguientes instrumentos: test de inteligencias múltiples, que describe 55 conductas clasificadas en once tipos de inteligencias; y el inventario de intereses profesionales de Angellini, que establece nueve áreas profesionales (Tabla $\mathrm{N}^{\circ} 1$ y Tabla $\mathrm{N}^{\circ} 2$ ).

Posteriormente, se procesó los datos a partir de una base de datos con el apoyo de un escáner de lectura de tarjetas ópticas. Se utilizó el software estadístico SPSS v19, para el uso de métodos estadísticos descriptivos e inferenciales.

\section{RESULTADOS}

De los 13459 alumnos, el 71,2\% son de colegios estatales y el $28,8 \%$, de colegios particulares; el $41,4 \%$ pertenece al cuarto año de secundaria y el 58,6\%, al quinto; el $53,9 \%$ están constituidos por mujeres y el $46,1 \%$, por varones.

Dentro de las dos áreas de interés profesional con mayores preferencias está, el área de ciencias físicas $(34,6 \%)$, donde el 27,5\% son mujeres y el $42,8 \%$, varones; $y$, el área de ciencias biológicas (16,8\%), en el que el $18,2 \%$ son mujeres y el $15,2 \%$, varones. El área persuasiva es el de menor preferencia $(4,5 \%)$ en los alumnos evaluados.

Las inteligencias que más destacan en ellos son la intrapersonal $(15,8 \%)$ y la lógicomatemática $(14,1 \%)$, mientras que las menos expresan son la religiosa- espiritual $(4,3 \%)$ y la sexual $(0,2 \%)$ (Tabla N ${ }^{\circ} 3$ y Tabla $\left.\mathrm{N}^{\circ} 4\right)$.

Los colegios estatales sobresalen en las inteligencias intrapersonal $(16,2 \%)$, naturalista o ecológica $(14,7 \%)$ y musical o rítmica $(12,7 \%)$; en tanto que los colegios particulares, en las inteligencias lógicomatemática $(18,9 \%)$, intrapersonal $(14,7 \%)$ y musical o rítmica $(13,6 \%)$.

En el caso del género (Tabla № 4), las mujeres desarrollan más las inteligencias intrapersonal $(18,9 \%)$, naturalista $\circ$ ecológica $(14,2 \%)$ y musical o rítmica
$(13,8 \%)$, a diferencia de los hombres que manifiestan más las inteligencias lógicomatemática $(21,3 \%)$, naturalista o ecológica $(13,2 \%)$ e intrapersonal $(12,2 \%)$.

Según la relación área profesional y tipo de inteligencia, los alumnos que eligieron el área artística (Arquitectura, Diseño Gráfico, Diseñador de Modas, Publicista) obtienen mayor puntuación en las inteligencias intrapersonal (15,4\%), musical o rítmica $(14,6)$ y naturalista $\circ$ ecológica (13,9\%); los que optaron por el área de cálculo (Matemática, Contabilidad, Banca y Finanzas, Comercio Exterior, Economía) expresan mayores inteligencias intrapersonal (15,9\%), lógico-matemática $(15,2 \%)$ y naturalista o ecológica $(12,9 \%)$; los que decidieron por el área de ciencias biológicas (Medicina Humana, Biología, Bioquímica y Farmacia, Enfermería, Tecnología Médica, Nutrición, Odontología) destacan en las inteligencias intrapersonal $(17,1 \%)$, naturalista o ecológica $(15,7 \%)$ y musical o rítmica $(12,3 \%)$.

Los del área de ciencias físicas (Ingeniería mecánica, Ingeniería civil, Ingeniería eléctrica, Ingeniería de sistemas e informática, Ingeniería de minas, Ingeniería ambiental, Ingeniería industrial) sobresalen en las inteligencias lógicomatemática (18\%), naturalista o ecológica e intrapersonal $(13,5 \%)$; mientras que los del área literaria (Periodismo, Literatura, Idiomas), en las inteligencias intrapersonal $(17,1 \%)$, naturalista o ecológica $(14,6 \%)$ y musical o rítmica $(13,3 \%)$.

Los estudiantes identificados con el área musical (Licenciatura en Arte, que comprende música, danza clásica y contemporánea) muestran mayor puntuación en las inteligencias musical o rítmica $(23,6 \%)$, intrapersonal $(13,7 \%)$ y naturalista o ecológica $(11,8 \%)$; los que prefieren el área de organización burocrática (Asistencia de gerencia, Contabilidad, Marketing, Administración, Ingeniería en agronegocios), reflejan mayores inteligencias intrapersonal (18\%), naturalista o ecológica $(14,9 \%)$ y lógicomatemática (13,8\%). 
Tabla Nº 3: Distribución de estudiantes evaluados según tipo de inteligencia por tipo de colegio.

\begin{tabular}{|c|c|c|c|c|}
\hline \multirow{2}{*}{\multicolumn{2}{|c|}{ Tipo de inteligencia }} & \multicolumn{2}{|c|}{ Tipo de colegio } & \multirow{2}{*}{ Total } \\
\hline & & Estatal & Particular & \\
\hline \multirow[t]{3}{*}{ Inteligencia humanista } & Evaluados & 505 & 185 & 690 \\
\hline & \% Tipo de inteligencia & $73,2 \%$ & $26,8 \%$ & $100,0 \%$ \\
\hline & \% Tipo de colegio & $5,3 \%$ & $4,8 \%$ & $5,1 \%$ \\
\hline \multirow[t]{3}{*}{ Inteligencia interpersonal } & Evaluados & 763 & 247 & 1010 \\
\hline & \% Tipo de inteligencia & $75,5 \%$ & $24,5 \%$ & $100,0 \%$ \\
\hline & $\%$ Tipo de colegio & $8,0 \%$ & $6,4 \%$ & $7,5 \%$ \\
\hline \multirow[t]{3}{*}{ Inteligencia intrapersonal } & Evaluados & 1551 & 567 & 2118 \\
\hline & $\%$ Tipo de inteligencia & $73,2 \%$ & $26,8 \%$ & $100,0 \%$ \\
\hline & \% Tipo de colegio & $16,2 \%$ & $14,7 \%$ & $15,8 \%$ \\
\hline \multirow{3}{*}{$\begin{array}{l}\text { Inteligencia kinestésica o } \\
\text { corporal }\end{array}$} & Evaluados & 951 & 390 & 1341 \\
\hline & \% Tipo de inteligencia & $70,9 \%$ & $29,1 \%$ & $100,0 \%$ \\
\hline & \% Tipo de colegio & $9,9 \%$ & $10,1 \%$ & $10,0 \%$ \\
\hline \multirow{3}{*}{$\begin{array}{l}\text { Inteligencia lógico } \\
\text { matemático }\end{array}$} & Evaluados & 1166 & 731 & 1897 \\
\hline & \% Tipo de inteligencia & $61,5 \%$ & $38,5 \%$ & $100,0 \%$ \\
\hline & $\%$ Tipo de colegio & $12,2 \%$ & $18,9 \%$ & $14,1 \%$ \\
\hline \multirow{3}{*}{$\begin{array}{l}\text { Inteligencia musical o } \\
\text { rítmica }\end{array}$} & Evaluados & 1213 & 524 & 1737 \\
\hline & \% Tipo de inteligencia & $69,8 \%$ & $30,2 \%$ & $100,0 \%$ \\
\hline & $\%$ Tipo de colegio & $12,7 \%$ & $13,6 \%$ & $12,9 \%$ \\
\hline \multirow{3}{*}{$\begin{array}{l}\text { Inteligencia naturalista } \\
\text { o ecológica }\end{array}$} & Evaluados & 1404 & 437 & 1841 \\
\hline & \% Tipo de inteligencia & $76,3 \%$ & $23,7 \%$ & $100,0 \%$ \\
\hline & $\%$ Tipo de colegio & $14,7 \%$ & $11,3 \%$ & $13,7 \%$ \\
\hline \multirow{3}{*}{$\begin{array}{l}\text { Inteligencia religiosa } \\
\text { y espiritual }\end{array}$} & Evaluados & 434 & 138 & 572 \\
\hline & \% Tipo de inteligencia & $75,9 \%$ & $24,1 \%$ & $100,0 \%$ \\
\hline & \% Tipo de colegio & $4,5 \%$ & $3,6 \%$ & $4,3 \%$ \\
\hline \multirow[t]{3}{*}{ Inteligencia sexual } & Evaluados & 21 & 2 & 23 \\
\hline & \% Tipo de inteligencia & $91,3 \%$ & $8,7 \%$ & $100,0 \%$ \\
\hline & $\%$ Tipo de colegio &, $2 \%$ &, $1 \%$ &, $2 \%$ \\
\hline \multirow{3}{*}{$\begin{array}{l}\text { Inteligencia verbal - } \\
\text { lingüística }\end{array}$} & Evaluados & 960 & 391 & 1351 \\
\hline & \% Tipo de inteligencia & $71,1 \%$ & $28,9 \%$ & $100,0 \%$ \\
\hline & \% Tipo de colegio & $10,0 \%$ & $10,1 \%$ & $10,1 \%$ \\
\hline \multirow{3}{*}{$\begin{array}{l}\text { Inteligencia visual - } \\
\text { espacial }\end{array}$} & Evaluados & 592 & 251 & 843 \\
\hline & \% Tipo de inteligencia & $70,2 \%$ & $29,8 \%$ & $100,0 \%$ \\
\hline & \% Tipo de colegio & $6,2 \%$ & $6,5 \%$ & $6,3 \%$ \\
\hline \multirow[t]{3}{*}{ Total } & Evaluados & 9560 & 3863 & 13423 \\
\hline & \% Tipo de inteligencia & $71,2 \%$ & $28,8 \%$ & $100,0 \%$ \\
\hline & $\%$ Tipo de colegio & $100,0 \%$ & $100,0 \%$ & $100,0 \%$ \\
\hline
\end{tabular}


Tabla N 4: Distribución de estudiantes evaluados según tipo de inteligencia por género.

\begin{tabular}{|c|c|c|c|c|}
\hline \multirow{2}{*}{\multicolumn{2}{|c|}{ Tipo de inteligencia }} & \multicolumn{2}{|c|}{ Género } & \multirow{3}{*}{$\begin{array}{r}\text { Total } \\
690\end{array}$} \\
\hline & & \multirow{2}{*}{$\frac{\text { Mujer }}{331}$} & \multirow{2}{*}{$\begin{array}{r}\text { Varón } \\
359\end{array}$} & \\
\hline Inteligencia humanista & Evaluados & & & \\
\hline & \% Tipo de inteligencia & $48,0 \%$ & $52,0 \%$ & $100,0 \%$ \\
\hline & \% Género & $4,6 \%$ & $5,8 \%$ & $5,1 \%$ \\
\hline \multirow[t]{3}{*}{ Inteligencia interpersonal } & Evaluados & 701 & 309 & 1010 \\
\hline & \% Tipo de inteligencia & $69,4 \%$ & $30,6 \%$ & $100,0 \%$ \\
\hline & \% Género & $9,7 \%$ & $5,0 \%$ & $7,5 \%$ \\
\hline \multirow[t]{3}{*}{ Inteligencia intrapersonal } & Evaluados & 1366 & 752 & 2118 \\
\hline & \% Tipo de inteligencia & $64,5 \%$ & $35,5 \%$ & $100,0 \%$ \\
\hline & \% Género & $18,9 \%$ & $12,2 \%$ & $15,8 \%$ \\
\hline \multirow{3}{*}{$\begin{array}{l}\text { Inteligencia kinestésica o } \\
\text { corporal }\end{array}$} & Evaluados & 651 & 690 & 1341 \\
\hline & \% Tipo de inteligencia & $48,5 \%$ & $51,5 \%$ & $100,0 \%$ \\
\hline & \% Género & $9,0 \%$ & $11,2 \%$ & $10,0 \%$ \\
\hline \multirow{3}{*}{$\begin{array}{l}\text { Inteligencia lógico } \\
\text { matemática }\end{array}$} & Evaluados & 582 & 1315 & 1897 \\
\hline & \% Tipo de inteligencia & $30,7 \%$ & $69,3 \%$ & $100,0 \%$ \\
\hline & \% Género & $8,0 \%$ & $21,3 \%$ & $14,1 \%$ \\
\hline \multirow{3}{*}{$\begin{array}{l}\text { Inteligencia musical o } \\
\text { rítmica }\end{array}$} & Evaluados & 1000 & 737 & 1737 \\
\hline & \% Tipo de inteligencia & $57,6 \%$ & $42,4 \%$ & $100,0 \%$ \\
\hline & \% Género & $13,8 \%$ & $11,9 \%$ & $12,9 \%$ \\
\hline \multirow{3}{*}{$\begin{array}{l}\text { Inteligencia naturalista } \\
\text { o ecológica }\end{array}$} & Evaluados & 1027 & 814 & 1841 \\
\hline & \% Tipo de inteligencia & $55,8 \%$ & $44,2 \%$ & $100,0 \%$ \\
\hline & \% Género & $14,2 \%$ & $13,2 \%$ & $13,7 \%$ \\
\hline \multirow{3}{*}{$\begin{array}{l}\text { Inteligencia religiosa } \\
\text { y espiritual }\end{array}$} & Evaluados & 329 & 243 & 572 \\
\hline & \% Tipo de inteligencia & $57,5 \%$ & $42,5 \%$ & $100,0 \%$ \\
\hline & \% Género & $4,5 \%$ & $3,9 \%$ & $4,3 \%$ \\
\hline \multirow[t]{3}{*}{ Inteligencia sexual } & Evaluados & 10 & 13 & 23 \\
\hline & \% Tipo de inteligencia & $43,5 \%$ & $56,5 \%$ & $100,0 \%$ \\
\hline & \% Género &, $1 \%$ &, $2 \%$ &, $2 \%$ \\
\hline \multirow{3}{*}{$\begin{array}{l}\text { Inteligencia verbal - } \\
\text { lingüística }\end{array}$} & Evaluados & 883 & 468 & 1351 \\
\hline & \% Tipo de inteligencia & $65,4 \%$ & $34,6 \%$ & $100,0 \%$ \\
\hline & \% Género & $12,2 \%$ & $7,6 \%$ & $10,1 \%$ \\
\hline \multirow{3}{*}{$\begin{array}{l}\text { Inteligencia visual - } \\
\text { espacial }\end{array}$} & Evaluados & 356 & 487 & 843 \\
\hline & \% Tipo de inteligencia & $42,2 \%$ & $57,8 \%$ & $100,0 \%$ \\
\hline & \% Género & $4,9 \%$ & $7,9 \%$ & $6,3 \%$ \\
\hline \multirow[t]{3}{*}{ Total } & Evaluados & 7236 & 6187 & 13423 \\
\hline & \% Tipo de inteligencia & $53,9 \%$ & $46,1 \%$ & $100,0 \%$ \\
\hline & \% Género & $100,0 \%$ & $100,0 \%$ & $100,0 \%$ \\
\hline
\end{tabular}


Tabla $N^{\circ}$ 5: Distribución de estudiantes evaluados por tipo de inteligencia según área vocacional.

\begin{tabular}{|c|c|c|c|c|c|c|c|c|c|c|c|}
\hline \multirow{2}{*}{\multicolumn{2}{|c|}{$\begin{array}{c}\text { Tipo de } \\
\text { Inteligencia }\end{array}$}} & \multicolumn{9}{|c|}{ Área } & \multirow{3}{*}{$\begin{array}{l}\text { Total } \\
680\end{array}$} \\
\hline & & \multirow{2}{*}{ Artíst. } & \multirow{2}{*}{$\begin{array}{r}\text { Cálculo } \\
61\end{array}$} & \multirow{2}{*}{$\begin{array}{r}\text { Cs. } \\
\text { Biológ. } \\
103\end{array}$} & \multirow{2}{*}{$\begin{array}{c}\text { Cs. } \\
\text { Físicas } \\
262\end{array}$} & \multirow{2}{*}{$\begin{array}{r}\text { Liter. } \\
54\end{array}$} & \multirow{2}{*}{$\frac{\text { Musical }}{34}$} & \multirow{2}{*}{$\begin{array}{r}\begin{array}{r}\text { Org. } \\
\text { Burocr. }\end{array} \\
38\end{array}$} & \multicolumn{2}{|c|}{ Persuas. Herv. Soc. } & \\
\hline Humanista & Evaluados & & & & & & & & 32 & 45 & \\
\hline & $\%$ Intelig. & $7,5 \%$ & $9,0 \%$ & $15,1 \%$ & $38,5 \%$ & $7,9 \%$ & $5,0 \%$ & $5,6 \%$ & $4,7 \%$ & $6,6 \%$ & $100,0 \%$ \\
\hline & \% Área & $5,6 \%$ & $5,5 \%$ & $4,6 \%$ & $5,7 \%$ & $4,8 \%$ & $4,3 \%$ & $5,2 \%$ & $5,3 \%$ & $4,0 \%$ & $5,1 \%$ \\
\hline \multirow[t]{3}{*}{ Interpersonal } & Evaluados & 72 & 109 & 182 & 289 & 68 & 54 & 55 & 52 & 117 & 998 \\
\hline & $\%$ Intelig. & $7,2 \%$ & $10,9 \%$ & $18, \%$ & $29,0 \%$ & $6,8 \%$ & $5,4 \%$ & $5,5 \%$ & $5,2 \%$ & $11,7 \%$ & $100,0 \%$ \\
\hline & \% Área & $7,9 \%$ & $9,8 \%$ & $8,2 \%$ & $6,3 \%$ & $6,0 \%$ & $6,8 \%$ & $7,5 \%$ & $8,7 \%$ & $10,3 \%$ & $7,6 \%$ \\
\hline \multirow[t]{3}{*}{ Intrapersonal } & Evaluados & 140 & 177 & 379 & 617 & 194 & 109 & 133 & 117 & 218 & 2084 \\
\hline & $\%$ Intelig. & $6,7 \%$ & $8,5 \%$ & $18,2 \%$ & $29,6 \%$ & $9,3 \%$ & $5,2 \%$ & $6,4 \%$ & $5,6 \%$ & $10,5 \%$ & $100,0 \%$ \\
\hline & \% Área & $15,4 \%$ & $15,9 \%$ & $17,1 \%$ & $13,5 \%$ & $17,1 \%$ & $13,7 \%$ & $18,0 \%$ & $19,5 \%$ & $19,3 \%$ & $15,8 \%$ \\
\hline \multirow[t]{3}{*}{ Kinestésica } & Evaluados & 106 & 93 & 229 & 471 & 128 & 77 & 61 & 51 & 115 & 1331 \\
\hline & $\%$ Intelig. & $8,0 \%$ & $7,0 \%$ & $17,2 \%$ & $35,4 \%$ & $9,6 \%$ & $5,8 \%$ & $4,6 \%$ & $3,8 \%$ & $8,6 \%$ & $100,0 \%$ \\
\hline & \% Área & $11,6 \%$ & $8,3 \%$ & $10,3 \%$ & $10,3 \%$ & $11,3 \%$ & $9,7 \%$ & $8,3 \%$ & $8,5 \%$ & $10,2 \%$ & $10,1 \%$ \\
\hline \multirow{3}{*}{$\begin{array}{l}\text { Lógico - } \\
\text { matemática }\end{array}$} & Evaluados & 109 & 170 & 265 & 822 & 140 & 88 & 102 & 76 & 94 & 1866 \\
\hline & $\%$ Intelig. & $5,8 \%$ & $9,1 \%$ & $14,2 \%$ & $44,1 \%$ & $7,5 \%$ & $4,7 \%$ & $5,5 \%$ & $4,1 \%$ & $5,0 \%$ & $100,0 \%$ \\
\hline & \% Área & $12,0 \%$ & $15,2 \%$ & $11,9 \%$ & $18,0 \%$ & $12,3 \%$ & $11,0 \%$ & $13,8 \%$ & $12,6 \%$ & $8,3 \%$ & $14,1 \%$ \\
\hline \multirow{3}{*}{$\begin{array}{l}\text { Musical o } \\
\text { rítmica }\end{array}$} & Evaluados & 133 & 133 & 273 & 504 & 151 & 188 & 95 & 70 & 172 & 1719 \\
\hline & $\%$ Intelig. & $7,7 \%$ & $7,7 \%$ & $15,9 \%$ & $29,3 \%$ & $8,8 \%$ & $10,9 \%$ & $5,5 \%$ & $4,1 \%$ & $10,0 \%$ & $100,0 \%$ \\
\hline & \% Área & $14,6 \%$ & $11,9 \%$ & $12,3 \%$ & $11,1 \%$ & $13,3 \%$ & $23,6 \%$ & $12,9 \%$ & $11,6 \%$ & $15,2 \%$ & $13,0 \%$ \\
\hline \multirow[t]{3}{*}{ Naturalista } & Evaluados & 127 & 144 & 348 & 614 & 166 & 94 & 110 & 66 & 144 & 1813 \\
\hline & $\%$ Intelig. & $7,0 \%$ & $7,9 \%$ & $19,2 \%$ & $33,9 \%$ & $9,2 \%$ & $5,2 \%$ & $6,1 \%$ & $3,6 \%$ & $7,9 \%$ & $100,0 \%$ \\
\hline & \% Área & $13,9 \%$ & $12,9 \%$ & $15,7 \%$ & $13,5 \%$ & $14,6 \%$ & $11,8 \%$ & $14,9 \%$ & $11,0 \%$ & $12,7 \%$ & $13,7 \%$ \\
\hline \multirow{3}{*}{$\begin{array}{l}\text { Religiosa y } \\
\text { espiritual }\end{array}$} & Evaluados & 30 & 54 & 99 & 211 & 42 & 32 & 34 & 22 & 37 & 561 \\
\hline & $\%$ Intelig. & $5,3 \%$ & $9,6 \%$ & $17,6 \%$ & $37,6 \%$ & $7,5 \%$ & $5,7 \%$ & $6,1 \%$ & $3,9 \%$ & $6,6 \%$ & $100,0 \%$ \\
\hline & \% Área & $3,3 \%$ & $4,8 \%$ & $4,5 \%$ & $4,6 \%$ & $3,7 \%$ & $4,0 \%$ & $4,6 \%$ & $3,7 \%$ & $3,3 \%$ & $4,2 \%$ \\
\hline \multirow[t]{3}{*}{ Sexual } & Evaluados & 1 & 4 & 6 & 7 & 1 & 0 & 4 & 0 & 0 & 23 \\
\hline & \% Intelig. & $4,3 \%$ & $17,4 \%$ & $26,1 \%$ & $30,4 \%$ & $4,3 \%$ &, $0 \%$ & $17,4 \%$ &, $0 \%$ &, $0 \%$ & $100,0 \%$ \\
\hline & \% Área &, $1 \%$ &, $4 \%$ &, $3 \%$ &, $2 \%$ &, $1 \%$ &, $0 \%$ &, $5 \%$ &, $0 \%$ &, $0 \%$ &, $2 \%$ \\
\hline \multirow{3}{*}{$\begin{array}{l}\text { Verbal - } \\
\text { lingüística }\end{array}$} & Evaluados & 90 & 106 & 221 & 435 & 111 & 72 & 68 & 78 & 124 & 1305 \\
\hline & $\%$ Intelig. & $6,9 \%$ & $8,1 \%$ & $16,9 \%$ & $33,3 \%$ & $8,5 \%$ & $5,5 \%$ & $5,2 \%$ & $6,0 \%$ & $9,5 \%$ & $100,0 \%$ \\
\hline & \% Área & $9,9 \%$ & $9,5 \%$ & $9,9 \%$ & $9,5 \%$ & $9,8 \%$ & $9,0 \%$ & $9,2 \%$ & $13,0 \%$ & $11,0 \%$ & $9,9 \%$ \\
\hline \multirow{6}{*}{$\begin{array}{l}\text { Visual - } \\
\text { espacial }\end{array}$} & Evaluados & 53 & 65 & 117 & 328 & 80 & 49 & 37 & 37 & 65 & 831 \\
\hline & $\%$ Intelig. & $6,4 \%$ & $7,8 \%$ & $14,1 \%$ & $39,5 \%$ & $9,6 \%$ & $5,9 \%$ & $4,5 \%$ & $4,5 \%$ & $7,8 \%$ & $100,0 \%$ \\
\hline & \% Área & $5,8 \%$ & $5,8 \%$ & $5,3 \%$ & $7,2 \%$ & $7,0 \%$ & $6,1 \%$ & $5,0 \%$ & $6,2 \%$ & $5,7 \%$ & $6,3 \%$ \\
\hline & Evaluados & 912 & 1116 & 2222 & 4560 & 1135 & 797 & 737 & 601 & 1131 & 13211 \\
\hline & $\%$ Intelig. & $6,9 \%$ & $8,4 \%$ & $16,8 \%$ & $34,5 \%$ & $8,6 \%$ & $6,0 \%$ & $5,6 \%$ & $4,5 \%$ & $8,6 \%$ & $100,0 \%$ \\
\hline & \% Área & $100,0 \%$ & $100,0 \%$ & $100,0 \%$ & $100,0 \%$ & $100,0 \%$ & $100,0 \%$ & $100,0 \%$ & $100,0 \%$ & $100,0 \%$ & $100,0 \%$ \\
\hline
\end{tabular}


Los que quieren dedicarse al área persuasiva (Ciencias de la Comunicación, Derecho, Diplomacia) desarrollan con mayor énfasis las inteligencias intrapersonal (19,5\%), verbal-lingüística (13\%) y lógicomatemática $(12,6 \%)$; y los que gustan del área de servicio social humanitaria (Psicología, Trabajo Social, Pedagogía), reflejan inteligencias intrapersonal $(19,3 \%)$, musical o rítmica $(15,2)$ y naturalista $\circ$ ecológica $(12,7 \%)$ (Tabla №5).

De acuerdo con la prueba estadística no paramétrica de Chi cuadrado, existe el 1\% de significancia en la relación entre el área vocacional y las inteligencias múltiples, el género y el tipo de colegio (estatal/ particular). También podemos determinar que hay relación entre las inteligencias múltiples y los tipos de colegios (estatal/ particular) y el género de los alumnos del cuarto y quinto de educación secundaria.

\section{DISCUSIÓN}

Elegir una carrera profesional en los jóvenes implica experimentar diversas experiencias personales, familiares, sociales y académicas en medio de un proceso complejo (1) y constante con la finalidad de decidir y optar un estilo de vida. Para esto será importante identificar y reconocer las distintas habilidades que los jóvenes han desarrollado y reforzado en su entorno desde épocas tempranas (2). Precisamente Gardner, en su teoría de las inteligencias múltiples asume a la inteligencia como la capacidad de afrontar situaciones problemáticas e incluso permite crear productos valiosos, los cuales son útiles para un contexto cultural (17). Este marco teórico, ha sido útil para nuestra investigación desarrollada en los alumnos del cuarto y quinto de educación secundaria de los colegios estatales y particulares, donde podemos evidenciar la existencia de diversos estilos y formas de confrontar situaciones.

De acuerdo con los resultados de la investigación, determinamos que los alumnos de colegios estatales han desarrollado la inteligencia intrapersonal $(16,2 \%)$, mientras que los alumnos de colegios particulares, la inteligencia lógico matemático $(18,9 \%)$. Este caso puede obedecer a que los jóvenes estén influenciados y reforzados por el medio donde se encuentran. También es importante que los alumnos reconozcan e integren sus diversos estilos de inteligencias para contar con mayores recursos al momento de resolver situaciones problemáticas en su futura formación profesional y de este modo puedan prevenirse deserciones $y$ frustraciones académicas (3). De modo que puedan ir construyendo y fortaleciendo un conjunto de habilidades que materialicen sus estilos de inteligencias y les permitan desempeñarse en su contexto profesional.

Afirmamos que lo más importante en la elección de una carrera profesional será reconocer la peculiaridad de los jóvenes en cuanto a su constitución intrínseca (personalidad y género) (15). Esta peculiaridad encontramos en la presente investigación puesto que los varones presentan mayor interés por carreras profesionales relacionadas con las ciencias físicas $(42,8 \%)$ y mayor desarrollo en la inteligencia lógico-matemática (21,3\%); mientras que las mujeres presentan mayor interés por carreras profesionales relacionadas con las ciencias biológicas $(18,2 \%)$ y mayor desarrollo en la inteligencia intrapersonal (18,9\%). Entonces advertimos que la individualidad promueve en los jóvenes la utilización de recursos psicológicos, los cuales son importantes para que logren identificar, desarrollar y direccionar sus estrategias personales, de modo que sean útiles y permitan dar mayor consistencia a su perfil profesional futuro (16).

Reconocemos que los jóvenes podrán desempeñarse en un contexto laboral exigente que implicará un estilo de vida (4); siempre y cuando logren poner en práctica sus diversas formas de aprendizaje (5). Por esta razón, es importante que la elección de carreras profesionales guarde relación con 
las inteligencias desarrolladas y reforzadas en el tiempo en el entorno familiar, social y académico, de tal modo que permitan direccionar una mejor decisión al momento de elegir una carrera durante los procesos de orientación vocacional y profesional.

Así, podemos decir que los jóvenes que eligen carreras relacionadas con el área de ciencias físicas (Ingeniería Mecánica, Ingeniería Civil, Ingeniería Eléctrica, Ingeniería de Sistemas e Informática, Ingeniería de Minas, Ingeniería Ambiental, Ingeniería Industrial) requieren de la inteligencia lógico-matemática; y los que eligen carreras relacionadas con el área persuasiva (Ciencias de la Comunicación, Derecho, Diplomacia) y el área de servicio social humanitaria (Psicología, Trabajo Social, Pedagogía), necesitan de la inteligencia musical para su futuro desempeño.

Advertimos, entonces, que existe la necesidad de identificar y promover distintas inteligencias en los jóvenes, pero focalizándolas en aquellas que son comunes a todas las carreras profesionales. En este sentido encontramos en los resultados que todas las carreras de las distintas áreas tienden a desarrollar habilidades direccionadas al razonamiento y resolución de problemas (inteligencia lógicomatemática, 14,1\%), a distinguir y utilizar elementos de su entorno (inteligencia naturalista, 13,7\%), y a identificar, asociar y distinguir los estímulos perceptuales del medio (inteligencia musical, 13\%).

A pesar de que los jóvenes deben desarrollar sus inteligencias múltiples de acuerdo con su carrera profesional, no obstante también deben tener un buen autoconcepto y autoconocimiento (19), para que puedan percibir una imagen de veracidad y precisión de sí mismos, de modo tal que puedan adecuarse a la realidad en sus decisiones vocacionales. En tal sentido la investigación muestra que los jóvenes tienen ese potencial, lo que es positivo porque podrían adecuar sus decisiones al contexto situacional, inteligencia intrapersonal (15,8\%). Para el conocimiento de sí mismos se sugiere tomar en cuenta la estructura de las cuatro dimensiones humanas (12), biológico, emocional, social y cognitivo, ya que permite trabajar las diversas inteligencias múltiples (8) y promover el equilibrio de la personalidad (11), la direccionalidad de su identidad (10) y la orientación de las necesidades personales e intereses vocacionales de los jóvenes; los cuales son importantes para darles consistencia al perfil profesional y laboral (13).

Asimismo, se deben considerar aquellas inteligencias que no logran desarrollarse con facilidad, ya que dentro del contexto socioeconómico actual los jóvenes reciben un cúmulo de estímulos que no les permite dar consistencia a sus decisiones, actitudes, valores y habilidades (9), factores que deben reforzarse para que puedan tener sostenibilidad en el futuro (6). Es por esto último que los orientadores y las familias deben asumir un rol protagónico en el proceso vocacional (7), convirtiéndose en ejes vitales, y a través de estos protagonistas, se articulen y soporten las reales decisiones de los jóvenes.

Dicho lo anterior, podemos remitirnos a nuestra investigación y observar que la inteligencia espiritual $(4,3 \%)$ y la inteligencia sexual $(0,2 \%)$ se hayan disminuidas, lo que es preocupante porque son inteligencias necesarias, útiles y aplicables en todas las carreras profesionales. Estas inteligencias permitirán a los jóvenes tener una valoración real de sus roles (inteligencia sexual) y la aplicabilidad de valores que le den significado a su futuro desempeño profesional, así como mayor consistencia a la vocación profesional. Siguiendo a Lemos y Thomas (15), Noronha (10), Godoy (18), Ríos (1) y Aisenson (5), debemos replantearnos, por lo tanto, las estrategias de identificación y desarrollo de habilidades de los jóvenes que están decidiendo su futuro profesional y estilo de vida. No solo debemos aplicar cuestionarios vocacionales, porque éstos solo miden gustos y preferencias a nivel cognitivo.

En tal sentido podemos tomar como 
dirección de ruta aquellas prácticas y experiencias, que estos autores han planteado y experimentado, para que los orientadores y las familias asuman un rol de acompañamiento real en los procesos de orientación vocacional (21); y de este modo, puedan lograr identificar, matizar y desarrollar las diversas habilidades y estilos de aprendizaje con las que cuentan los jóvenes. Por supuesto que ello debe estar sustentado en una metodología pedagógica y psicológica a nivel individual y grupal (22), que permita potenciar y abrir espacios de investigación e implementación de programas educativos tanto en la enseñanza básica como a nivel superior.

De este modo podremos cambiar el paradigma de los procesos de orientación vocacional y profesional, para dirigirlos a un escenario de habilidades y competencias que permitan promover una vocación real, una mejor adaptabilidad a la realidad y sobre todo la demostración de valores, aspectos que son una necesidad y exigencia en las diversas áreas profesionales y en el competitivo mercado laboral.

\section{REFERENCIAS BIBLIOGRÁFICAS}

1. Ríos V. Estilos de personalidad en los procesos de orientación vocacional en alumnos del cuarto y quinto grado de secundaria: Apuntes de Ciencia \& Sociedad. Universidad Continental. $2011 ; 1(1): 3-8$.

2. De los Santos M. Efectos de un programa tutorial en la elección ocupacional. Chiclayo: Publicaciones: Poemario; 2006.

3. Vicuña L. Elaboración del inventario de intereses vocacionales para carreras técnicas y de oficio. Revista de investigación en psicología. 2002; 5(1): 15-19.

4. Ríos V, Supo L. Influencia de los hábitos de estudio en la elección de carreras profesionales en alumnos del cuarto y quinto grado de secundaria: Apuntes de Ciencia \& Sociedad. Universidad Continental. 2011; 1(2): 92-99.

5. Aisenson D, et al. Aprendizaje, sujetos y escenarios. Buenos Aires: Novedades Educativas; 2007.

6. De León $\mathrm{T}$, et al. La orientación vocacional y la modificación del criterio de pre elección de carrera técnica. México DF: Educere; 2005.

7. Cano MA. Motivación y elección de carrera, Revista Mexicana de Orientación Educativa. 2008; 5 (13): 6-9.

8. Castro C, Egaña V. Adolescentes y elección de carrera profesional: Factores que influyen en la decisión. Santiago de Chile: Colectivo latinoamericano de jóvenes; 2009.

9. Frisancho A. La orientación vocacional en los colegios públicos y privados de Lima. Revista de Psicología de la Universidad Nacional Mayor de San Marcos. 2006; 9(1):23-35.

10. Noronha A, et al. Las correlaciones entre los intereses vocacionales y de inteligencia en los adolescentes. São Paulo: Universidad de San Francisco. 2009; $11(2): 114-128$.

11. Josemberg $M$, et al. El proceso de orientación profesional antes de que 
el siglo XXI: perspectivas y desafíos. Brasilia. Universidad federal de paraíba; 2002.

12. Ríos V. Entrenamiento y Desarrollo de habilidades en las organizaciones. Huancayo: Fondo Editorial Continental; 2009.

13. Rehbein L, Martínez G, Rose I, Gloria C. Los estilos epistémicos y tipos de personalidad como factores asociados a la elección de carrera: Revista de Pedagogía. Universidad Central de Venezuela. 2009; 30(86): 11 5-134.

14. Valdés V. Orientación profesional. México: Pearson educación; 2004.

15. Aisenson D, Figari C, et al. Orientación vocacional: Proyectos de vida, adquisición de recursos personales y trabajo. Ensayos y experiencias Colección psicología y educación. Novedades Educativas. Buenos Aires: Tomo $\mathrm{n}^{\circ}$ 28. 1999.

16. Ramos M, Delgado G, Barrenechea O. Orientación Educativa en la Escuela: Perspectivas para la psicología en el Perú: Revista peruana de Psicología. Colegio de Psicólogos del Perú. 2007; 1(1): 66-74.

17. Gardner H. Frames of mind: the theory of multiple intelligences. Basic Books. New York; 1983.

18. Godoy S, et al. Los instrumentos de la inteligencia y el interés en la orientación profesional. Sao Paulo; Universidad de San Francisco; 2008.

19. Schmidt A. Autoconcepto y motivaciones vocacionales en estudiantes de primer año de carreras de Humanidades y Ciencias Exactas. Paraná. Universidad Católica de Argentina; 2010.

20. Noronha A, Matteo A, Rodolfo A. Orientación profesional: análisis de la producción científica. Itatiba. Universidad de San Francisco. 2006; 11 (1): 75-84.

21. Gan F, Soto R. Carrera profesional: claves, competencias y vitaminas. España. Ediciones Díaz de Santos; 2007.

22. Machado L. Retos actuales de la educación técnico profesionales. Orientación profesional. España. Santillana; 2012. 